EPJ Web of Conferences 41, 05034 (2013)

DOI: $10.1051 /$ epjconf/20134105034

(C) Owned by the authors, published by EDP Sciences, 2013

\title{
Two-dimensional electronic femtosecond stimulated Raman spectroscopy
}

\author{
D. E. Wilcox and J. P. Ogilvie \\ Department of Physics and Biophysics, University of Michigan, 450 Church Street, Ann Arbor, MI, \\ USA 4810
}

\begin{abstract}
We report two-dimensional electronic spectroscopy with a femtosecond stimulated Raman scattering probe. The method reveals correlations between excitation energy and excited state vibrational structure following photoexcitation. We demonstrate the method in rhodamine $6 \mathrm{G}$.
\end{abstract}

Two-dimensional electronic spectroscopy (2DES) has emerged as an attractive method for studying the ultrafast dynamics and electronic structure of a wide range of condensed matter systems [1]. Femtosecond stimulated Raman spectroscopy (FSRS) [2-5] has provided an incisive structural probe of the primary motions in vision [6], proton transfer in GFP [7] and other charge transfer processes [8]. Here we combine these two methods, demonstrating 2DES with a FSRS probe (2DE-FSRS).

We have previously demonstrated pulse-shaping-based 2DES in the pump probe geometry [9], enabling the straightforward use of a continuum probe to expand the frequency information available in 2DES [10]. This versatile setup also allows the straightforward use of more sophisticated probes such as FSRS. In a standard FSRS experiment, a single actinic pump pulse is used. Thus there is no information available about the excitation frequency dependence of the observed FSRS signal. In 2DE-FSRS the actinic pump is comprised of a pair of pulses, separated by delay $t_{1}$. A time $t_{2}$ following the actinic pulse sequence, a combination of narrowband Raman pump and continuum probe initiate stimulated Raman scattering. A differential measurement is made (actinic pump on and off) to obtain the FSRS modes excited as a result of the actinic pump. As in 2DES, Fourier transforming with respect to the $t_{l}$ delay resolves the excitation frequency, allowing correlations to be made between excitation and detection frequencies. Recording different 2DE-FSRS spectra as a function of waiting time $t_{2}$ will show how these correlations evolve.

The pulse sequence and experimental setup for 2DE-FSRS are shown in Figure 1A. Part of the output of a regenerative amplifier (Spitfire Pro, $800 \mathrm{~nm}, 1 \mathrm{~mJ}$ pulses at $1 \mathrm{kHz}$ ) is used to pump a noncollinear optical parametric amplifier (NOPA) [11] to provide the actinic pump. The NOPA pulse is sent into an acousto-optic pulse-shaper (Dazzler, Fastlite), giving near transform limited pulses of 25 fs, centered at $530 \mathrm{~nm}$. In addition to providing pulse compression, the Dazzler was used to create a pair of actinic pump pulses with variable delay $t_{l}$. A continuum probe pulse is created by focusing a small fraction of the $800 \mathrm{~nm}$ pulse into a $2 \mathrm{~mm}$ thick sapphire window with a $200 \mathrm{~mm}$ focal length lens and collimated using a $250 \mathrm{~mm}$ spherical mirror. The Raman pump is obtained by spectrally filtering a portion of the $800 \mathrm{~nm}$ light with a combination of notch filters to produce $\sim 1 \mathrm{~nm}$ pulses. The relative polarization of all pump and probe beams are parallel. A linear stage was used to generate the $t_{2}$ delay between the actinic pump pulses and the FSRS probe pulses. The pump beam was focused with a $250 \mathrm{~mm}$ fused silica lens to a spot size of $200 \mu \mathrm{m}$ in a $300 \mu \mathrm{m}$ thick sample cell.

This is an Open Access article distributed under the terms of the Creative Commons Attribution License 2.0, which permits unrestricted use, distribution, and reproduction in any medium, provided the original work is properly cited. 
The probe beam was focused with a $250 \mathrm{~mm}$ spherical mirror to a spot size of $100 \mu \mathrm{m}$ and overlapped in the sample with the pump beam at a crossing angle of $\sim 2^{\circ}$. These focusing and excitation conditions produced a $\sim 70 \%$ excitation level of the sample. Cross-correlation between the pump and probe pulses indicated a time resolution of $\sim 200 \mathrm{fs}$. The pump beam was electronically "chopped" at $500 \mathrm{~Hz}$ by the Dazzler, and the probe beam was spectrally resolved at $1 \mathrm{kHz}$ using a Horiba Jobin Yvon iHR320 spectrometer and Pixis 100B CCD camera. We note that in the experimental configuration shown here, both 2DES and 2DE-FSRS spectra are obtained simultaneously, as the Raman pump pulse is chopped.

2DE-FSRS data taken on rhodamine 6G in ethanol is shown in Figure 1B, for a waiting time $t_{2}=$ $300 \mathrm{fs}$. Along the detection frequency axis, several excited state Raman modes are clearly observed. In this system, we did not observe time dependent shifts of the observed modes, or excitation wavelength dependent features in the FSRS spectrum over the excitation wavelength range that was studied.
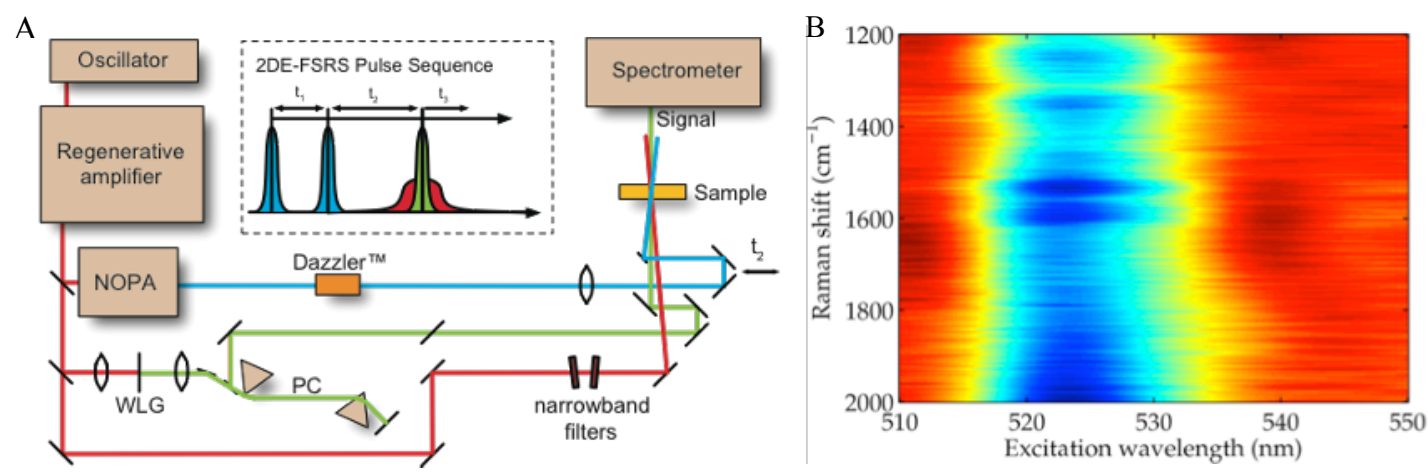

Fig 1: A: Experimental setup for 2DE-FSRS. (NOPA: noncollinear optical parametric amplifier, WLG: white light generation in a sapphire plate, PC: prism compressor) B: 2DE-FSRS spectrum of rhodmamine $6 \mathrm{G}$ in ethanol at a waiting time of $300 \mathrm{fs}$.

By resolving the excitation frequency dependence of FSRS without compromising time resolution, 2DE-FSRS promises to provide a structural probe of excited states as they evolve. We note that the FSRS probe, being non-resonant, can access optically dark states such as charge transfer states that are invisible to 2DES and other resonant spectroscopies. In addition the broadband FSRS probe offers an attractive alternative to the relatively narrowband spectral data obtained in experiments using IR pulses. The combination of 2DES and 2DE-FSRS will provide complementary electronic and vibrational signatures of spectroscopic intermediates, facilitating their identification.

\section{Acknowledgements:}

This material is based upon work supported as part of the Center for Solar and Thermal Energy Conversion, an Energy Frontier Research Center funded by the U. S. Department of Energy, Office of Science, Office of Basic Energy Sciences under Award Number DE-SC0000957.

\section{References:}

1. M. H. Cho, "Coherent two-dimensional optical spectroscopy," Chemical Reviews 108, 1331-1418 (2008).

2. M. Yoshizawa, Y. Hattori, and T. Kobayashi, "Femtosecond time-resolved resonance Raman gain spectroscopy in polydiacetylene," Physical Review B 49, 13259-13262 (1994). 
3. M. Yoshizawa and M. Kurosawa, "Femtosecond time-resolved Raman spectroscopy using stimulated Raman scattering," Physical Review A 61, art. no.-013808 (2000).

4. D. W. McCamant, P. Kukura, S. Yoon, and R. A. Mathies, "Femtosecond broadband stimulated Raman spectroscopy: Apparatus and methods," Review of Scientific Instruments 75, 4971-4980 (2004).

5. D. W. McCamant, P. Kukura, and R. A. Mathies, "Femtosecond time-resolved stimulated Raman spectroscopy: Application to the ultrafast internal conversion in beta-carotene," Journal of Physical Chemistry A 107, 8208-8214 (2003).

6. P. Kukura, D. W. McCamant, S. Yoon, D. B. Wandschneider, and R. A. Mathies, "Structural observation of the primary isomerization in vision with femtosecond-stimulated Raman," Science 310, 1006-1009 (2005).

7. C. Fang, R. R. Frontiera, R. Tran, and R. A. Mathies, "Mapping GFP structure evolution during proton transfer with femtosecond Raman spectroscopy," Nature 462, 200-U274 (2009).

8. J. V. Lockard, A. B. Ricks, D. T. Co, and M. R. Wasielewski, "Interrogating the Intramolecular Charge-Transfer State of a Julolidine-Anthracene Donor-Acceptor Molecule with Femtosecond Stimulated Raman Spectroscopy," Journal of Physical Chemistry Letters 1, 215-218 (2010).

9. J. A. Myers, K. L. M. Lewis, P. F. Tekavec, and J. P. Ogilvie, "Two-color two-dimensional Fourier transform electronic spectroscopy with a pulse-shaper," Optics Express 16, 1742017428 (2008).

10. P. F. Tekavec, J. A. Myers, K. L. M. Lewis, and J. P. Ogilvie, "Two dimensional electronic spectroscopy with a continuum probe," Optics Letters 34, 1390-1392 (2009).

11. T. Wilhelm, J. Piel, and E. Riedle, "Sub-20-fs pulses tunable across the visible from a bluepumped single-pass noncollinear parametric converter," Optics Letters 22, 1494-1496 (1997). 